\title{
The prognostic value of USP14 and PSMD14 expression in non- small cell lung cancer
}

\author{
Jian Lei ${ }^{1,2}$, Xiaoliu Liu ${ }^{3}$, Wei Liu ${ }^{2}$, Yun Zhang ${ }^{2}$, Zhihong Liu ${ }^{2}$ \\ ${ }^{1}$ Affiliated Cancer Hospital and Institute of Guangzhou Medical University, Guangzhou Municipal and Guangdong Provincial Key Laboratory \\ of Protein Modification and Degradation, State Key Laboratory of Respiratory Disease, School of Basic Medical Sciences, Guangzhou Medical \\ University, Guangzhou, China; ${ }^{2}$ Department of Pathology, Hunan Cancer Hospital and The Affiliated Cancer Hospital of Xiangya School of \\ Medicine, Central South University, Changsha, China; ${ }^{3}$ Department of Hematology and Oncology, The Fourth Hospital of Changsha and The \\ Affiliated Changsha hospital of Hunan Normal University, Changsha, China \\ Contributions: (I) Conception and design: J Lei, W Liu; (II) Administrative support: Z Liu; (III) Provision of study materials or patients: J Lei, Y \\ Zhang; (IV) Collection and assembly of data: J Lei; (V) Data analysis and interpretation: J Lei, X Liu; (VI) Manuscript writing: All authors; (VII) \\ Final approval of manuscript: All authors. \\ Correspondence to: Jian Lei. Affiliated Cancer Hospital and Institute of Guangzhou Medical University, Guangzhou Municipal and Guangdong \\ Provincial Key Laboratory of Protein Modification and Degradation, State Key Laboratory of Respiratory Disease, School of Basic Medical Sciences, \\ Guangzhou Medical University, Guangzhou 511436, China; Department of Pathology, Hunan Cancer Hospital and The Affiliated Cancer Hospital \\ of Xiangya School of Medicine, Central South University, Changsha 410013, China. Email: leijian@hnca.org.cn.
}

Background: Ubiquitin specific peptidase 14 (USP14) and proteasome 26S subunit, non-ATPase 14 (PSMD14) are two deubiquitinases that are closely related to the human 19S proteasome. These are highly expressed in various types of cancers and are associated with prognosis. However, the expression, clinicopathological features, and prognostic relevance of these two deubiquitinases remain unclear in patients with non-small cell lung cancer (NSCLC). Moreover, the correlation between the expression of these two deubiquitinases in NSCLC has not been reported.

Methods: In this study, the expression of USP14 and PSMD14 in NSCLC tissues and adjacent non-tumor tissues were examined by immunohistochemical staining. The association of these two deubiquitinases with the clinicopathological features and overall survival (OS) of patients with NSCLC was evaluated meanwhile.

Results: The expression of USP14 and PSMD14 was upregulated in NSCLC tissues compared with adjacent non-tumor tissues. High expression of both these deubiquitinases was positively correlated with the TNM stage of NSCLC. In addition, PSMD14 was positively correlated with lymph node metastasis in NSCLC. The survival analysis showed that elevated levels of USP14 or PSMD14 were associated with poorer survival of NSCLC patients compared with low expression of USP14 or PSMD14. Cox regression analysis indicated that TNM stage, USP14, and PSMD14 were independent prognostic factors for OS in NSCLC.

Conclusions: This study demonstrated that USP14 and PSMD14 may play important roles in the progression of NSCLC, especially when they are expressed simultaneously at elevated levels. Thus, USP14 and PSMD14 may be potential novel biomarkers and therapeutic targets for the prognosis and treatment of patients with NSCLC.

Keywords: Non-small cell lung cancer (NSCLC); ubiquitin specific peptidase 14 (USP14); proteasome 26S subunit, non-ATPase 14 (PSMD14); deubiquitinase; combined expression

Submitted May 10, 2021. Accepted for publication Jun 16, 2021.

doi: 10.21037/atm-21-2748

View this article at: https://dx.doi.org/10.21037/atm-21-2748 


\section{Introduction}

Lung cancer is the most common malignant tumor with the highest morbidity and mortality in China and world-wide $(1,2)$. China has the largest number of newly diagnosed lung cancer cases and related deaths, accounting for one-third of the total global cases $(3,4)$. Non-small cell lung cancer (NSCLC) is the main pathological type of lung cancer and can be classified into squamous cell carcinoma (SCC) and adenocarcinoma. The primary treatments for NSCLC are surgery, chemotherapy, and radiotherapy. In recent years, molecular targeted therapy and immunotherapy have shown increasing potential. Despite continuous improvements in traditional and emerging treatments, the 5-year overall survival (OS) rate of NSCLC patients is still relatively low, at about $16 \%(5-7)$. Therefore, the search for novel tumor markers and effective drug targets is of great significance for the early diagnosis, precise treatment, and prognosis of patients with NSCLC.

The ubiquitin-proteasome pathway (UPP) is the main pathway for protein degradation in eukaryotic organisms $(8,9)$. Deubiquitinase can rescue the substrate protein from degradation by removing ubiquitin (10). Moreover, deubiquitinases may function as oncogenes or tumor suppressor genes to participate in the occurrence and development of tumors. They can also affect the development of tumors by regulating the activity of tumorrelated proteins (11-13). Ubiquitin specific peptidase 14 (USP14; also known as ubp6) and proteasome 26S subunit, non-ATPase 14 (PSMD14; also known as POH1 or rpn11), are important deubiquitinases that are closely related to the human 19S proteasome. They are highly expressed in many tumors and are associated with cancer prognosis $(14,15)$. However, to date, there has been a paucity of reports investigating USP14 and PSMD14 in lung cancer, including NSCLC. Moreover, there have been no reports about the expression of these two proteins in lung SCC, nor the clinicopathological significance and prognostic significance of the combined expression of these two proteins in NSCLC.

In this study, the protein expression of USP14 and PSMD14 was analyzed in 184 samples of NSCLC tissues (including lung SCC and adenocarcinoma). The correlation between the expression of USP14 and PSMD14 in NSCLC was examined. Subsequently, the association of USP14 and PSMD14 with the clinicopathological features and OS of NSCLC patients was evaluated. Our investigation suggested that USP14 and PSMD14 may serve as potential prognostic markers and therapeutic targets for the treatment of patients with NSCLC.

We present the following article in accordance with the REMARK reporting checklist (available at https://dx.doi. org/10.21037/atm-21-2748).

\section{Methods}

\section{Tissue specimens}

A total of 184 NSCLC tissues and corresponding adjacent non-cancerous tissues were obtained from the Department of Pathology in the Hunan Cancer Hospital, China. All specimens were fixed with formaldehyde and embedded with paraffin. All procedures performed in this study involving human participants were in accordance with the Declaration of Helsinki (as revised in 2013). The study was approved by Medical ethics committee of Hunan Cancer Hospital (No. 29 of scientific research quick review in 2021), obtaining exemption of informed consent. Patients did not receive radiotherapy or chemotherapy prior to collection of samples.

\section{Clinicopathological evaluation and follow-up}

Tissues were classified into the histological subtypes according to the classification of lung cancer by the World Health Organization in 2015. Samples were also classified according to age, gender, history of tobacco smoking, degree of tumor differentiation, tumor size, lymph node station, and TNM stage. All patients were followed up from the day of surgery. The median follow-up time was 44 months. Smoking history was defined as smoking at least one cigarette per day for more than half a year. OS was calculated from the date of surgery to the date of death or the last follow-up date.

\section{Immunobistochemical staining}

Human NSCLC tissues and adjacent non-cancerous tissues were cut into $4 \mu \mathrm{m}$ slices. After baking at $70{ }^{\circ} \mathrm{C}$ for 30 minutes, the slices were dewaxed and hydrated using a xylene and alcohol series, respectively. Endogenous peroxidase activity was blocked by incubation with $3 \%$ hydrogen peroxide at room temperature for 10 minutes. Antigen recovery was performed by heating with a pressure cooker in citric acid antigen repair solution ( $\mathrm{pH}$ 6.0). After blocking with $10 \%$ goat serum, the slices were incubated with rabbit anti-PSMD14 monoclonal antibody (ab109123) 

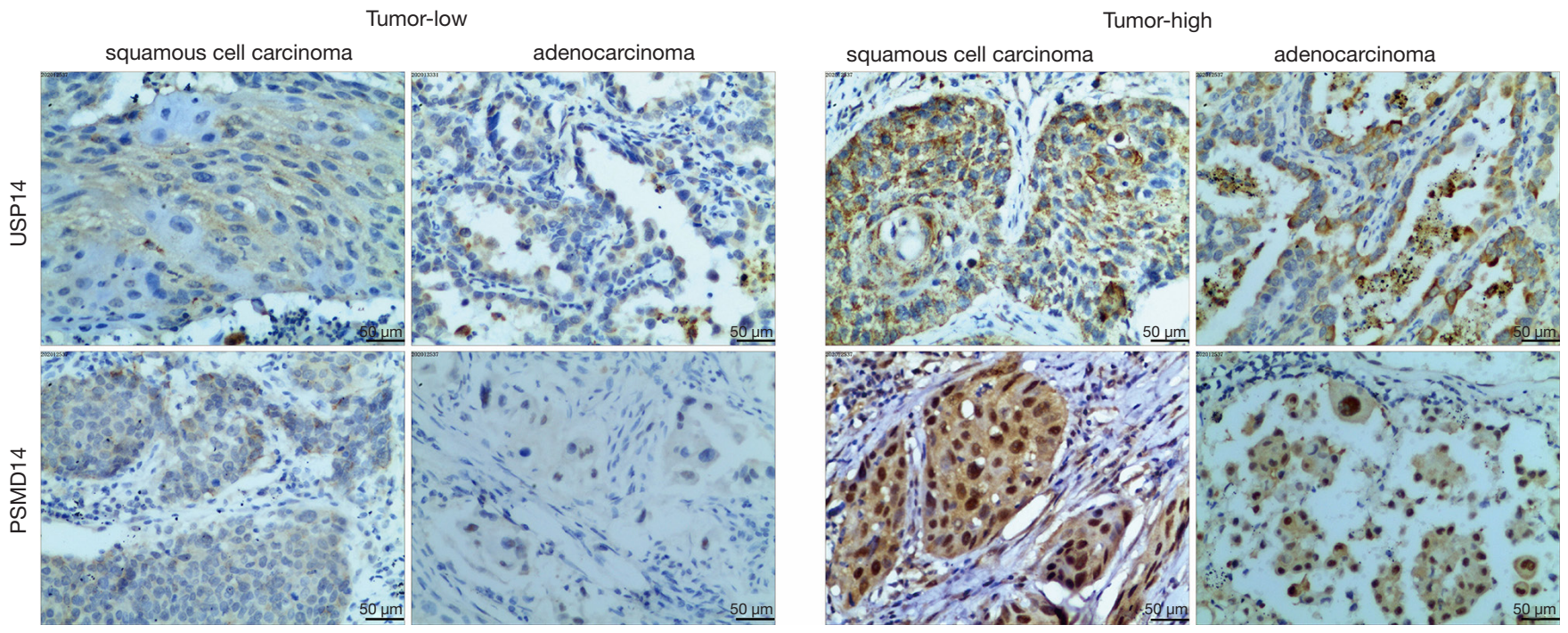

Figure 1 Immunostaining of USP14 and PSMD14 in NSCLC tissues. The scale bar represents $50 \mu m$. USP14, ubiquitin specific peptidase 14; PSMD14, proteasome 26S subunit, non-ATPase 14; NSCLC, non-small cell lung cancer.

or USP14 polyclonal antibody (ab71165; Abcam, Cambridge, UK) at $4{ }^{\circ} \mathrm{C}$ overnight. The tissue sections were then washed in phosphate-buffered saline (PBS) and incubated with a horseradish peroxidase-conjugated secondary antibody (MaixinBiol, Fuzhou, China) for 1 hour at room temperature. Samples were stained with diaminobenzidine and counterstained with hematoxylin according to the MaxVision kit (MaixinBiol) instructions.

\section{Assessing immunobistochemical staining}

The immunohistochemical results were independently evaluated by two experienced pathologists and scored according to Friedrich's criteria. The staining intensity of tumor cells was evaluated with a score of 0 (negative), 1 (mild), 2 (moderate), or 3 (strong). The proportion of positive tumor cells was evaluated with a score of $0(0-5 \%)$, $1(6-20 \%), 2(21-50 \%)$, or $3(51-100 \%)$. The final score was obtained by multiplying the scores of the staining intensity and the proportion of positive cells. A score $\leq 1$ was considered low expression, and a score $>1$ was considered high expression.

\section{Statistical analysis}

All statistical analyses were performed by SPSS version 20.0 (IBM, Chicago, IL, USA). The relationships between clinicopathological parameters and USP14 or PSMD14 expression were analyzed by the $\chi^{2}$ or Fisher's exact test. The OS distribution was calculated by the Kaplan-Meier method and analyzed by the log-rank test. Univariate and multivariate analyses were based on the Cox proportional hazard regression model. $\mathrm{P}$ value $<0.05$ was considered statistically significant.

\section{Results}

The expression of USP14 and PSMD14 in buman NSCLC tissues

To observe the expression of USP14 and PSMD14 in NSCLC tissues, immunohistochemical staining was performed on 184 NSCLC tissue samples and the corresponding adjacent non-cancerous tissues. USP14 immunostaining was located in the cytoplasm of tumor cells. In contrast, PSMD14 immunostaining was primarily detected in the cytoplasm of tumor cells and partially in the nuclei of tumor cells. USP14 and PSMD14 expression were both significantly upregulated in NSCLC tissues compared to the corresponding adjacent non-cancerous tissues (Figure 1 and Table 1).

\section{Association of the expression of USP14 and PSMD14 with clinicopathological characteristics in NSCLC}

USP14 expression was significantly associated with TNM stage $(\mathrm{P}=0.020$, Table 2$)$. In addition, PSMD14 was obviously 
Table 1 The expression of USP14 and PSMD14 in NSCLC tissues

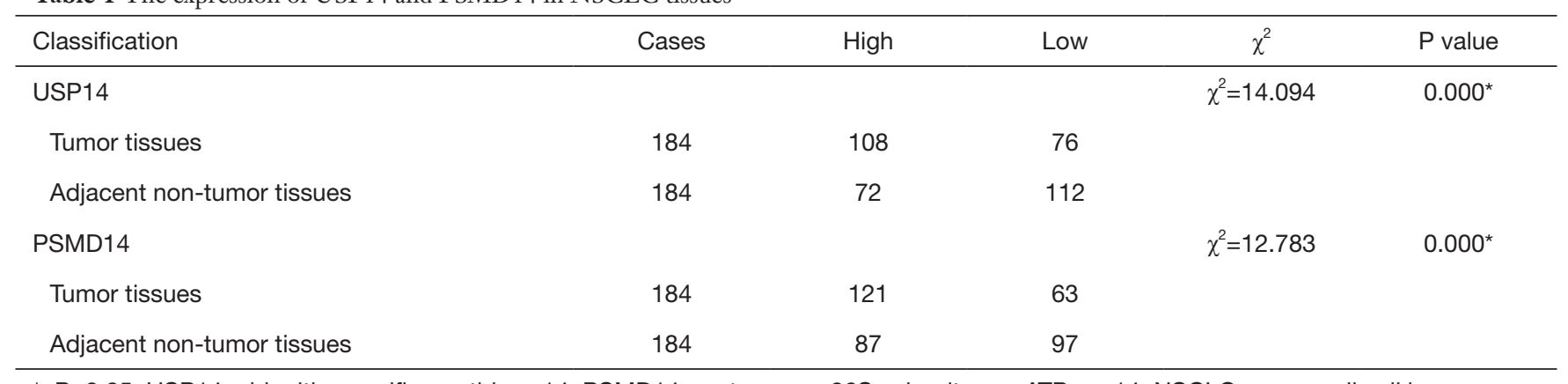

*, P<0.05. USP14, ubiquitin specific peptidase 14; PSMD14, proteasome 26 S subunit, non-ATPase 14; NSCLC, non-small cell lung cancer.

correlated with lymph node metastasis $(\mathrm{P}=0.027)$, as well as TNM stage $(\mathrm{P}=0.019)$ in NSCLC (Table 3). However, there was no association between PSMD14 expression and histological subtype, age, gender, tobacco smoking history, tumor size, nor degree of tumor differentiation. Similarly, there was no association between USP14 expression and the other clinicopathological characteristics. These results suggested that high expression of USP14 and PSMD14 were both associated with the severity and progression of NSCLC.

The association between clinicopathological characteristics and the combined expression of USP14 and PSMD14 in NSCLC was assessed (Table 4). There were 78 cases of high USP14 and high PSMD14 expression, 30 cases of high USP14 and low PSMD14 expression, 33 cases of low USP14 and low PSMD14 expression, and 43 cases of low USP14 and high PSMD14 expression. Samples with high USP14 and high PSMD14 expression profiles showed significant differences in histological subtype, age, lymph node metastasis, and TNM stage compared to samples in the other three expression profile subgroups. However, there were no significant differences in gender, tobacco smoking history, tumor size, degree of tumor differentiation, lymph node metastasis, and TNM stage among these latter expression profile subgroups. In addition, there was no obvious difference in any of the clinicopathological features between the high USP14 and low PSMD14 subgroup and the low USP14 and high PSMD14 subgroup. These results further suggested that USP14 and PSMD14 may play important roles in the progression of NSCLC, especially when they are concurrently expressed at high levels.

\section{The association between $O S$ and the expression of USP14 and PSMD14 in non-small cell lung carcinoma}

The prognostic significance of USP14 and PSMD14 expression in NSCLC patients was assessed by the KaplanMeier survival analysis and log-rank test. The median survival time of patients with high USP14 expression was 39 months, and 58 months in patients with low USP14 expression. In addition, the median survival time of patients with high PSMD14 expression was 36 months, and 59 months in patients with low PSMD14 expression. The survival analysis showed that high expression of USP14 and high expression PSMD14 were both associated with poorer survival of NSCLC patients compared with low expression of USP14 and low expression of PSMD14, respectively $(\mathrm{P}=0.001$; Figure $2 A, B)$.

The combined expression of USP14 and PSMD14 was assessed in terms of the OS of patients with NSCLC. Compared with the subgroup expressing low USP14 and low PSMD14, the other three subgroups were all associated with shorter OS in NSCLC patients $(\mathrm{P}<0.001$; Figure $2 C)$. However, there was no noticeable difference in the OS among these latter three subgroups.

\section{Univariate and multivariate Cox regression analyses of prognostic factors of survival in NSCLC}

Univariate and multivariate Cox proportional hazard regression analyses were performed to investigate the influence of each clinicopathological feature on the OS in NSCLC patients. Univariate analyses demonstrated that age $(\mathrm{P}=0.023)$, lymph node metastasis $(\mathrm{P}<0.001)$, TNM stage $(\mathrm{P}<0.001)$, degree of differentiation $(\mathrm{P}<0.001)$, USP14 expression $(\mathrm{P}<0.001)$, and $\mathrm{PSMD} 14$ expression $(\mathrm{P}=0.001)$ were all correlated with shorter OS in NSCLC patients (Table 5). Multivariate analyses showed that TNM stage $(\mathrm{P}<0.001)$, USP14 expression $(\mathrm{P}=0.014)$, and PSMD14 expression $(\mathrm{P}=0.020)$ were independent prognostic factors for OS in NSCLC patients (Table 6). 
Table 2 The expression of USP14 was correlated with clinicopathological features in NSCLC patients

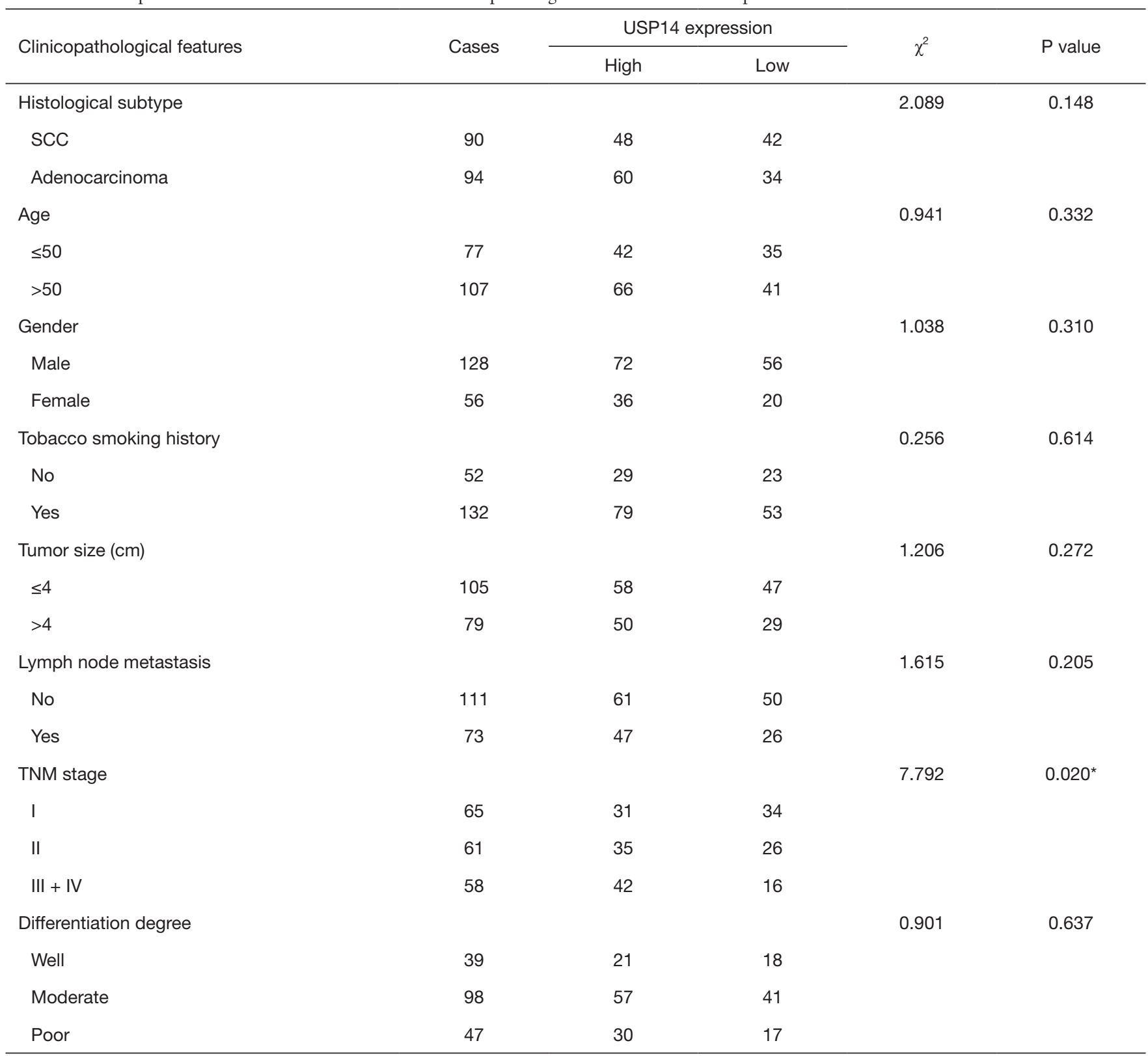

*, P<0.05. USP14, ubiquitin specific peptidase 14; NSCLC, non-small cell lung cancer; SCC, squamous cell carcinoma.

\section{Discussion}

Compared with small cell lung cancer, NSCLC is characterized by low malignancy, slow progression, and late metastasis. However, at diagnosis, about $50 \%$ of patients are in the middle and late stages of the disease, and at this stage, surgery is generally difficult and complete removal of the tumor is rare. Moreover, postoperative recurrence and metastasis can often occur $(6,7)$. Therefore, accurate methods for early diagnosis and effective treatment of lung cancer are urgently needed.

Ubiquitin specific proteases (USP) belong to the cysteine protease family, which contains more than 50 kinds of deubiquitin enzymes. The members of this family all contain a highly conserved USP domain. The coding gene of USP14 is located on human chromosome 18p11.32, which consists of 494 amino acids $(16,17)$. The role of 
Table 3 The expression of PSMD14 was correlated with clinicopathological features in NSCLC patients

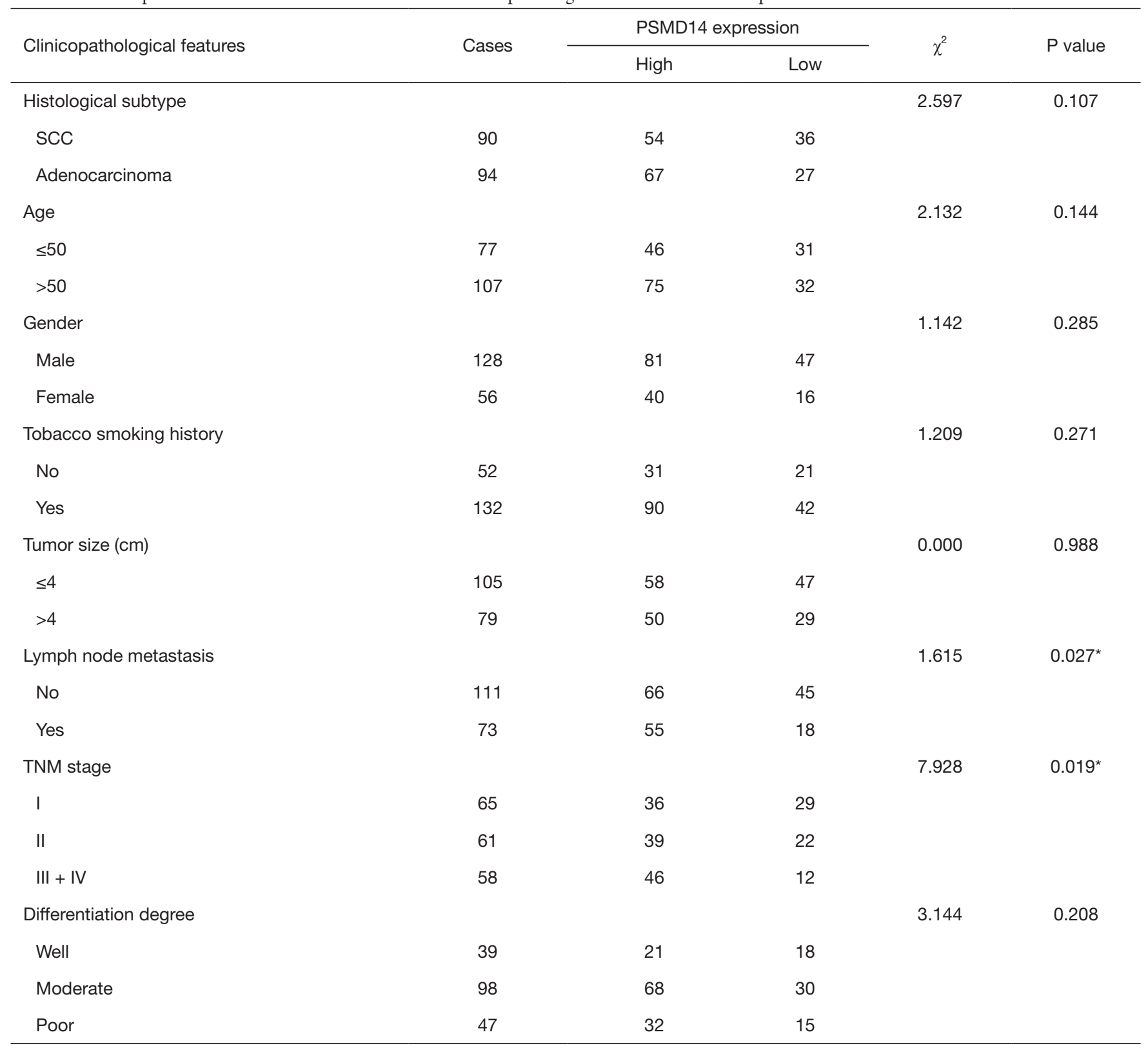

*, P<0.05. PSMD14, proteasome 26 S subunit, non-ATPase 14; NSCLC, non-small cell lung cancer; SCC, squamous cell carcinoma.

USP14 in various tumors has been widely studied (18). Shinji et al. (19) found that USP14 expression was not only increased in colorectal cancer cell lines, but also correlated with TNM stage, lymph node, and liver metastasis in colorectal cancer patients. Moreover, the OS of patients with high USP14 expression was poor in colorectal cancer patients. Wu and colleagues (20) reported elevated expression of USP14 in the tissues of lung adenocarcinoma compared with the corresponding adjacent non-cancerous tissues. At the same time, follow-up studies showed that patients with high USP14 expression had shorter OS and poorer prognosis. USP14 has also been shown to be highly expressed in ovarian cancer, endometrial cancer, breast cancer, hepatocellular carcinoma, pancreatic ductal adenocarcinoma, oral cancer, esophageal cancer, gastric cancer, and other tumor tissues, with most patients showing poor prognosis $(15,21-27)$.

JAMMs is a family of deubiquitinating enzymes with 
Table 4 The combined expression of USP14 and PSMD14 was correlated with clinicopathological features in NSCLC patients

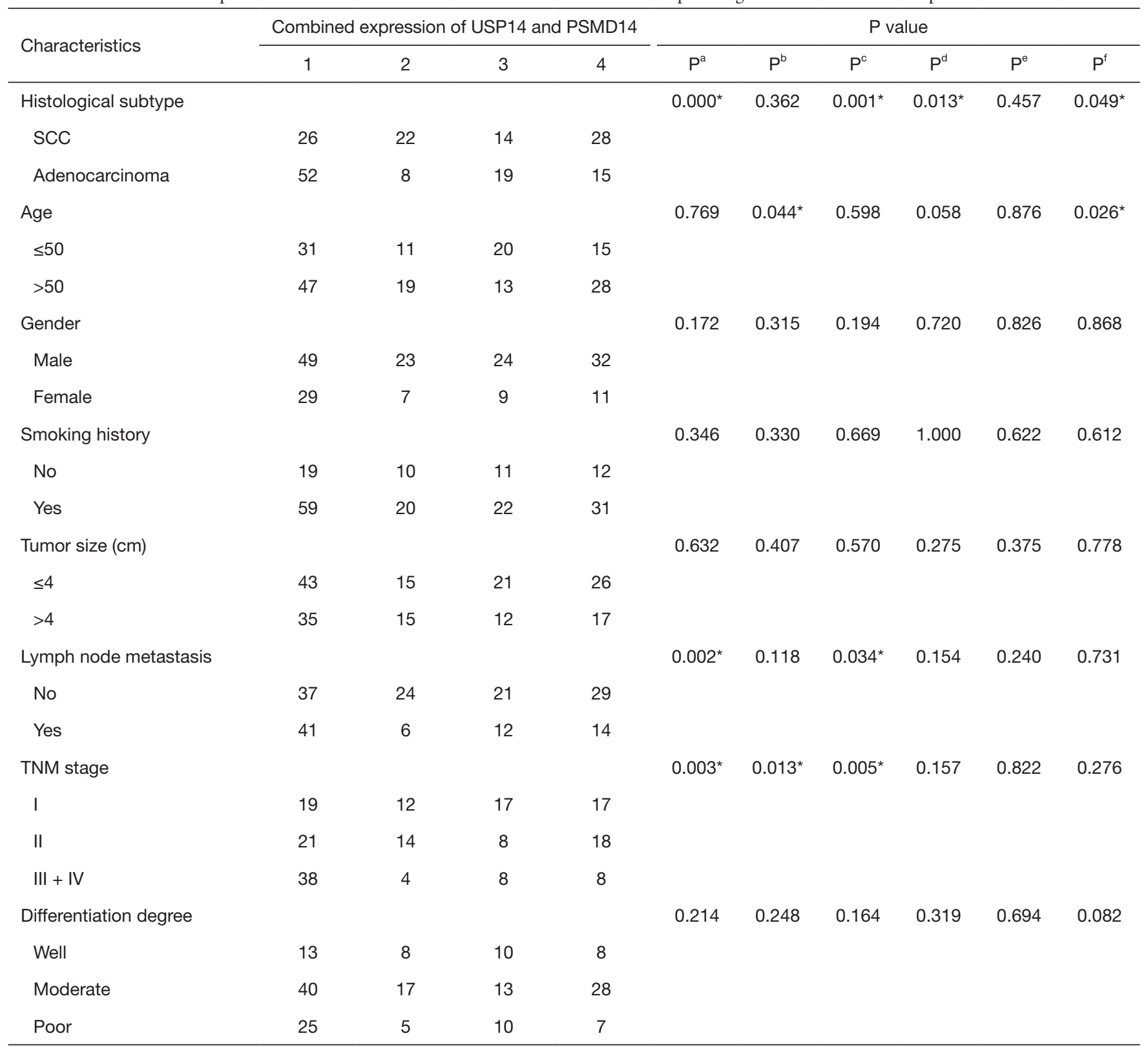

1, high USP14 + high PSMD14; 2, high USP14 + low PSMD14; 3, low USP14 + low PSMD14; 4, low USP14 + high PSMD14; Pa, 1 vs. 2; $\mathrm{P}^{\mathrm{b}}, 1$ vs. 3; $\mathrm{P}^{\mathrm{c}}, 1$ vs. 4; $\mathrm{P}^{\mathrm{d}}, 2$ vs. $3 ; \mathrm{P}^{\mathrm{e}}, 2$ vs. $4 ; \mathrm{P}^{\mathrm{f}}, 3$ vs. 4. ${ }^{*}, \mathrm{P}<0.05$. USP14, ubiquitin specific peptidase 14; PSMD14, proteasome 26S subunit, non-ATPase 14; NSCLC, non-small cell lung cancer; SCC, squamous cell carcinoma.

metalloproteinase activity. One member of this family, namely, PSMD14, is a component of the $19 \mathrm{~S}$ regulatory particle lid structure, which acts as a rate-limiting enzyme during the degradation of substrate proteins by the proteasome. The gene encoding PSMD14 is located on human chromosome 2q24.2, which consists of 310 amino acids $(8,28,29)$. PSMD14 has been shown to play an important role in tumorigenesis and development. Gene expression across normal and tumor tissues (GENT) confirmed that PSMD14 mRNA expression was increased in bladder cancer, breast cancer, cervical cancer, liver cancer, esophageal cancer, colon cancer, renal cancer, lung cancer, ovarian cancer, thyroid cancer, testicular cancer, prostate cancer, and other tumor tissues (30,31). Wang et al. reported 
A

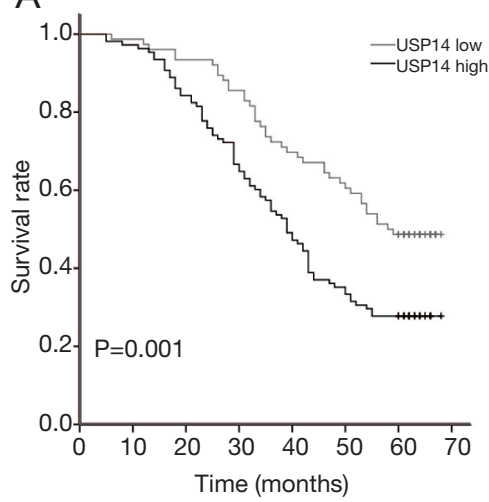

B

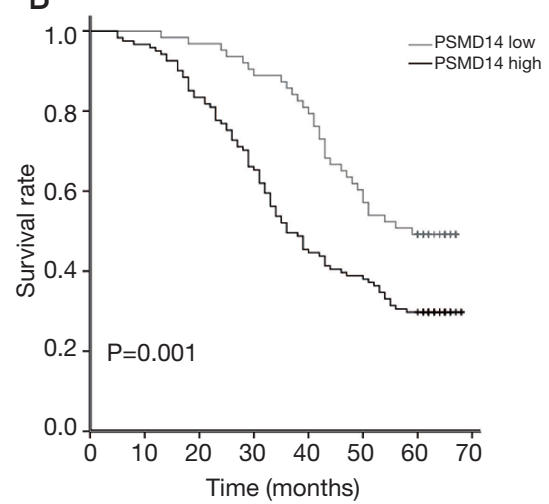

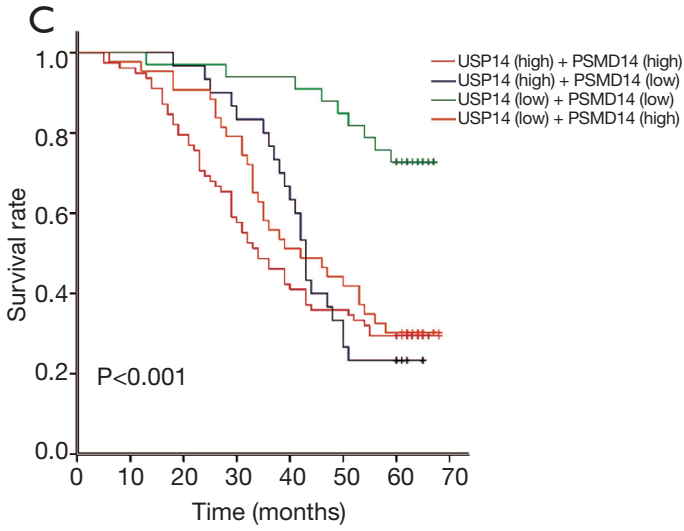

Figure 2 Kaplan-Meier survival curves of USP14 and PSMD14 expression for OS in patients with non-small cell lung carcinoma. (A) The prognostic value of low and high USP14 expression was measured by Kaplan-Meier survival curves. (B) The prognostic value of low and high PSMD14 expression was measured by Kaplan-Meier survival curves. (C) The prognostic value of combined expression of USP14 and PSMD14 was measured by Kaplan-Meier survival curves. USP14, ubiquitin specific peptidase 14; PSMD14, proteasome 26S subunit, nonATPase 14; OS, overall survival.

Table 5 Univariate Cox regression analysis of prognostic factors of survival in NSCLC patients

\begin{tabular}{lcc}
\hline Characteristics & Hazard ratio $(95 \%$ Cl) & P value \\
\hline Histological subtype & $0.908(0.757-1.089)$ & 0.300 \\
Age & $1.536(1.060-2.226)$ & $0.023^{*}$ \\
Gender & $1.064(0.720-1.571)$ & 0.756 \\
Tobacco smoking history & $1.157(0.770-1.737)$ & 0.482 \\
Tumor size (cm) & $1.252(0.869-1.802)$ & 0.227 \\
Lymph node metastasis & $3.522(2.430-5.105)$ & $<0.001^{*}$ \\
TNM stage & $2.470(1.947-3.134)$ & $<0.001^{*}$ \\
Degree of differentiation & $0.405(0.239-0.688)$ & $<0.001^{*}$ \\
USP14 expression & $1.914(1.301-2.816)$ & $<0.001^{*}$ \\
PSMD14 expression & $1.967(1.308-2.957)$ & $0.001^{*}$ \\
\hline
\end{tabular}

*, $\mathrm{P}<0.05$. NSCLC, non-small cell lung cancer; Cl, confidence interval; USP14, ubiquitin specific peptidase 14; PSMD14, proteasome 26S subunit, non-ATPase 14.

that high PSMD14 expression was related with poor prognosis of hepatocellular carcinoma, esophageal cancer, and colorectal cancer. Down regulation of PSMD14 protein expression by RNA interference inhibited the growth of these three tumor types and promoted the apoptosis of cancer cells (32). Other reports have also confirmed that elevated PSMD14 expression is not only related to poor prognosis of esophageal cancer patients, but is also related to the disease progression of multiple myeloma and enhanced invasion of breast cancer cells (33-35).
Although many studies have investigated the relationship between the expression of USP14 or PSMD14 and various tumor tissues, to our knowledge, there have been no reports examining the relationship between lung SCC and USP14 or PSMD14. Moreover, the clinicopathological significance of the combined expression of these two proteins in NSCLC has not been investigated. This current study examined tissues from 90 patients with SCC. Expression of USP14 and PSMD14 were both significantly upregulated in NSCLC tissues compared to the corresponding adjacent 
Table 6 Multivariate Cox regression analysis of prognostic factors of survival in non-small cell lung carcinoma patients

\begin{tabular}{lcc}
\hline Characteristics & Hazard ratio $(95 \%$ Cl) & P value \\
\hline Age & $0.772(0.523-1.139)$ & 0.193 \\
Degree of differentiation & $0.791(0.441-1.419)$ & 0.431 \\
Lymph node metastasis & $1.695(0.916-3.137)$ & 0.093 \\
TNM stage & $2.809(1.702-4.635)$ & $<0.001^{*}$ \\
USP14 expression & $1.747(1.119-2.728)$ & $0.014^{*}$ \\
PSMD14 expression & $1.679(1.086-2.596)$ & $0.020^{*}$ \\
\hline
\end{tabular}

*, $\mathrm{P}<0.05$. Cl, confidence interval; USP14, ubiquitin specific peptidase 14; PSMD14, proteasome $26 \mathrm{~S}$ subunit, non-ATPase 14.

non-cancerous tissues. High expression of USP14 was significantly associated with late TNM stage, while elevated PSMD14 expression was significantly correlated with lymph node metastasis as well as late TNM stage. These results are consistent with previous reports $(20-25,34,35)$ showing that PSMD14 or USP14 are associated with clinicopathologic features in other tumors. Furthermore, there were significant differences in histological subtype, age, lymph node metastasis, and TNM stage between patients in the high USP14 and high PSMD14 expression subgroup compared to patients in the other expression profile subgroups. Compared with the single protein expression of USP14 or PSMD14, we divided the combined expression of USP14 and PSMD14 into four groups and obtained more meaningful indicators. For example, high USP14 and high PSMD14 expression were more common in people older than 50 years old, while low USP14 and low PSMD14 expression were more common in people younger than 50 years old. The survival analyses showed that high expression of USP14 or PSMD14 were both positively associated with shorter OS of NSCLC patients. NSCLC patients in the low PSMD14 and low USP14 expression subgroup had longer OS compared with to patients in the other expression profile subgroups. Cox proportional hazard model demonstrated that TNM stage, USP14, and PSMD14 were independent prognostic factors of shorter OS in NSCLC patients.

As this was a retrospective cohort study with a limited sample size, there were certain limitations to this investigation. Future studies should expand the sample size and collate other detailed clinicopathological information and follow-up data to further verify these conclusions.

\section{Conclusions}

This investigation demonstrated that expression of USP14 and PSMD14 were both significantly upregulated in NSCLC tissues. High expression of USP14 and PSMD14 were both associated with the severity and progression of NSCLC. USP14 and PSMD14 may play important roles in the progression of NSCLC, especially when both are concurrently expressed at elevated levels. USP14 and PSMD14 may be potential novel biomarkers and therapeutic targets for evaluating prognosis and clinical treatment of patients with NSCLC.

\section{Acknowledgments}

Funding: None.

\section{Footnote}

Reporting Checklist: The authors have completed the REMARK reporting checklist. Available at https:// dx.doi.org/10.21037/atm-21-2748

Data Sharing Statement: Available at https://dx.doi. org/10.21037/atm-21-2748

Conflicts of Interest: All authors have completed the ICMJE uniform disclosure form (available at https://dx.doi. org/10.21037/atm-21-2748). The authors have no conflicts of interest to declare.

Ethical Statement: The authors are accountable for all aspects of the work in ensuring that questions related to the accuracy or integrity of any part of the work are appropriately investigated and resolved. All procedures performed in this study involving human participants were in accordance with the Declaration of Helsinki (as revised in 2013). The study was approved by Medical ethics committee of Hunan Cancer Hospital (No. 29 of scientific 


\section{Page 10 of 11}

research quick review in 2021), obtaining exemption of informed consent.

Open Access Statement: This is an Open Access article distributed in accordance with the Creative Commons Attribution-NonCommercial-NoDerivs 4.0 International License (CC BY-NC-ND 4.0), which permits the noncommercial replication and distribution of the article with the strict proviso that no changes or edits are made and the original work is properly cited (including links to both the formal publication through the relevant DOI and the license). See: https://creativecommons.org/licenses/by-nc-nd/4.0/.

\section{References}

1. Ettinger DS. Ten years of progress in non-small cell lung cancer. J Natl Compr Canc Netw 2012;10:292-5.

2. Alexander M, Kim SY, Cheng H. Update 2020: management of non-small cell lung cancer. Lung 2020;198:897-907.

3. Chen $\mathrm{W}$, Zheng R, Baade PD, et al. Cancer statistics in China, 2015. CA Cancer J Clin 2016;66:115-32.

4. Chen WQ, Li H, Sun KX, et al. Report of cancer incidence and mortality in China, 2014. Zhonghua Zhong Liu Za Zhi 2018;40:5-13.

5. Reck M, Rabe KF. Precision diagnosis and treatment for advanced non-small-cell lung cancer. $\mathrm{N}$ Engl J Med 2017;377:849-61.

6. Schiller JH. A new standard of care for advanced lung cancer. N Engl J Med 2018;378:2135-7.

7. Kowalczyk A, Jassem J. Multidisciplinary team care in advanced lung cancer. Transl Lung Cancer Res 2020;9:1690-8.

8. Bard JAM, Goodall EA, Greene ER, et al. Structure and function of the $26 \mathrm{~S}$ proteasome. Annu Rev Biochem 2018;87:697-724.

9. Livneh I, Cohen-Kaplan V, Cohen-Rosenzweig C, et al. The life cycle of the $26 \mathrm{~S}$ proteasome: from birth, through regulation and function, and onto its death. Cell Res 2016;26:869-85.

10. Lee MJ, Lee BH, Hanna J, et al. Trimming of ubiquitin chains by proteasome-associated deubiquitinating enzymes. Mol Cell Proteomics 2011;10:R110.003871.

11. Huang $X$, Zhang $X, X u J$, et al. Deubiquitinating enzyme: a potential secondary checkpoint of cancer immunity. Front Oncol 2020;10:1289.

12. D'Arcy P, Brnjic S, Olofsson MH, et al. Inhibition of proteasome deubiquitinating activity as a new cancer
Lei et al. The prognostic value of USP14 and PSMD14 in NSCLC

therapy. Nat Med 2011;17:1636-40.

13. Hussain S, Zhang Y, Galardy PJ. DUBs and cancer: the role of deubiquitinating enzymes as oncogenes, non-oncogenes and tumor suppressors. Cell Cycle 2009;8:1688-97.

14. Tian Z, D'Arcy P, Wang X, et al. A novel small molecule inhibitor of deubiquitylating enzyme USP14 and UCHL5 induces apoptosis in multiple myeloma and overcomes bortezomib resistance. Blood 2014;123:706-16.

15. Zhu Y, Zhang Y, Sui Z, et al. USP14 de-ubiquitinates vimentin and miR-320a modulates USP14 and vimentin to contribute to malignancy in gastric cancer cells. Oncotarget 2017;8:48725-36.

16. Hu M, Li P, Song L, et al. Structure and mechanisms of the proteasome-associated deubiquitinating enzyme USP14. EMBO J 2005;24:3747-56.

17. Lee BH, Lee MJ, Park S, et al. Enhancement of proteasome activity by a small-molecule inhibitor of USP14. Nature 2010;467:179-84.

18. Lundgren S, Odrzywol E. USP14 inhibitors as potential anticancer agents. Future Med Chem 2018;10:1741-3.

19. Shinji $S$, Naito $Z$, Ishiwata $S$, et al. Ubiquitin-specific protease 14 expression in colorectal cancer is associated with liver and lymph node metastases. Oncol Rep 2006;15:539-43.

20. Wu N, Liu C, Bai C, et al. Over-expression of deubiquitinating enzyme USP14 in lung adenocarcinoma promotes proliferation through the accumulation of -catenin. Int J Mol Sci 2013;14:10749-60.

21. Wang Y, Wang J, Zhong J, et al. Ubiquitin-specific protease 14 (USP14) regulates cellular proliferation and apoptosis in epithelial ovarian cancer. Med Oncol 2015;32:379.

22. Huang G, Li L, Zhou W. USP14 activation promotes tumor progression in hepatocellular carcinoma. Oncol Rep 2015;34:2917-24.

23. Vogel RI, Pulver T, Heilmann W, et al. USP14 is a predictor of recurrence in endometrial cancer and a molecular target for endometrial cancer treatment. Oncotarget 2016;7:30962-76.

24. Zhang J, Zhang D, Sun L. Knockdown of ubiquitinspecific protease 14 (USP14) inhibits the proliferation and tumorigenesis in esophageal squamous cell carcinoma cells. Oncol Res 2017;25:249-57.

25. Chen X, Wu J, Chen Y, et al. Ubiquitin-specific protease 14 regulates cell proliferation and apoptosis in oral squamous cell carcinoma. Int J Biochem Cell Biol 2016;79:350-9. 
26. Hang C, Gong C, Fang Y, et al. Ubiquitin-specific protease 14 (USP14) promotes proliferation and metastasis in pancreatic ductal adenocarcinoma. J Mol Histol 2021;52:187-96.

27. Zhu L, Yang S, He S, et al. Downregulation of ubiquitinspecific protease 14 (USP14) inhibits breast cancer cell proliferation and metastasis, but promotes apoptosis. J Mol Histol 2016;47:69-80.

28. Rosenzweig R, Osmulski PA, Gaczynska M, et al. The central unit within the 19S regulatory particle of the proteasome. Nat Struct Mol Biol 2008;15:573-80.

29. Verma R, Aravind L, Oania R, et al. Role of Rpn11 metalloprotease in deubiquitination and degradation by the 26S proteasome. Science 2002;298:611-5.

30. Perez C, Li J, Parlati F, et al. Discovery of an inhibitor of the proteasome subunit Rpn11. J Med Chem 2017;60:1343-61. Erratum in: J Med Chem 2017;60:3217.

31. Shin G, Kang TW, Yang S, et al. GENT: gene expression

Cite this article as: Lei J, Liu X, Liu W, Zhang Y, Liu Z. The prognostic value of USP14 and PSMD14 expression in nonsmall cell lung cancer. Ann Transl Med 2021;9(12):1019. doi: 10.21037/atm-21-2748 database of normal and tumor tissues. Cancer Inform 2011;10:149-57.

32. Wang CH, Lu SX, Liu LL, et al. POH1 Knockdown Induces Cancer Cell Apoptosis via p53 and Bim. Neoplasia 2018;20:411-24.

33. Song Y, Li S, Ray A, et al. Blockade of deubiquitylating enzyme Rpn11 triggers apoptosis in multiple myeloma cells and overcomes bortezomib resistance. Oncogene 2017;36:5631-8.

34. Zhu R, Liu Y, Zhou H, et al. Deubiquitinating enzyme PSMD14 promotes tumor metastasis through stabilizing SNAIL in human esophageal squamous cell carcinoma. Cancer Lett 2018;418:125-34.

35. Luo G, Hu N, Xia X, et al. RPN11 deubiquitinase promotes proliferation and migration of breast cancer cells. Mol Med Rep 2017;16:331-8.

(English Language Editor: J. Teoh) 